Pacific Journal of Mathematics

THE NONCOMMUTATIVE TOPOLOGY OF
ONE-DIMENSIONAL SPACES 


\title{
THE NONCOMMUTATIVE TOPOLOGY OF ONE-DIMENSIONAL SPACES
}

\author{
TERRY A. LORING
}

If $X$ and $Y$ are compact topological spaces, the unital star-homomorphisms from $C(X)$ to $C(Y)$ satisfy certain homotopy properties when $X$ is an absolute neighborhood retract. We show that two of these properties still hold when $C(Y)$ is replaced by a "noncommutative space", i.e. an arbitrary unital $C^{*}$-algebra, but only under the additional assumption that $X$ is one-dimensional. Specifically, we show that $C(X)$ is semiprojective and that two unital star-homomorphisms from $C(X)$ to a $C^{*}$-algebra $A$ are homotopic whenever they are close.

Introduction. In topological shape theory, a space $P$ with bad local properties is realized as an inverse limit of absolute neighborhood retracts (ANR's). The reason for using ANR's is that continuous functions to ANR's have two nice homotopy properties. Let $X$ be a compact ANR. Two continuous functions from a space $Y$ to $X$ are homotopic whenever they are close in an appropriate sense [5, 1.5.3]. This rigidity result is used to show that a function from an inverse limit of spaces to $X$ can be deformed to factor through one of those spaces [5, 1.3.2]. From this follows the fact, essential to shape theory, that two inverse systems of ANR's which both have limit $P$ are equivalent in an appropriate way. Therefore, one may define the shape of $P$ as the equivalence class of these systems, and use a representative system to study the global properties of $P$.

The Gelfand transformation translates the above into homotopy results about either two homomorphisms from $C(X)$ to $A$ or a homomorphism from $C(X)$ to an inductive limit $\underset{\lim }{\longrightarrow} A_{n}$, where $A$ and $A_{n}$ are commutative unital $C^{*}$-algebras. Our main results, Theorems $\mathrm{A}$ and $\mathrm{B}$ below, show that these homotopy results still hold when $X$ is one-dimensional and the commutivity assumption on $A$ and $A_{n}$ is dropped. The requirement that $X$ be one-dimensional is necessary for Theorem A to hold in general. In [4], it was shown that Theorem A fails for the two-torus.

The question of whether Theorem B holds for the two-torus is equivalent to the following open problem. Roughly, it asks whether close commuting unitary pairs can be connected. 
Question. Does there exist $\varepsilon>0$ such that, for any $C^{*}$-algebra $A$ and unitaries $U_{0}, U_{1}, V_{0}, V_{1} \in A$ satisfying

$$
U_{0} V_{0}=V_{0} U_{0}, \quad U_{1} V_{1}=V_{1} U_{1}, \quad\left\|U_{0}-U_{1}\right\|<\varepsilon, \quad\left\|V_{0}-V_{1}\right\|<\varepsilon,
$$

there exist continuous unitary paths $U_{t}, V_{t}$ in $A$ from $U_{0}$ to $U_{1}$ and $V_{0}$ to $V_{1}$ satisfying

$$
U_{t} V_{t}=V_{t} U_{t} \text { for all } t \in[0,1]
$$

Unless otherwise stated, all $C^{*}$-algebras shall be unital, and all homomorphisms shall be unital star-homomorphisms.

Theorem A. Let $X$ be a one-dimensional finite $C W$-complex, $B=$ $\lim _{n} B_{n}$ an inductive limit of a sequence of $C^{*}$-algebras with not necessarily injective connecting maps, and $\varphi: C(X) \rightarrow B$ a homomorphism. Then there exists $N$ and a homomorphism $\bar{\varphi}: C(X) \rightarrow B_{N}$ such that the diagram

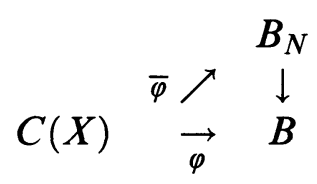

commutes up to homotopy.

THEOREM B. Let $X$ be a one-dimensional finite $C W$-complex. There exists $\varepsilon>0$ and $f_{1}, \ldots, f_{k} \in C(X)$ such that whenever $\varphi, \psi: C(X) \rightarrow B$ are two homomorphisms, from $C(X)$ to a $C^{*}$-algebra $B$, such that

$$
\left\|\varphi\left(f_{i}\right)-\psi\left(f_{i}\right)\right\|<\varepsilon, \quad i=1, \ldots, k,
$$

then $\varphi$ and $\psi$ are homotopic.

Effros and Kaminker [2] introduced the notion of a semiprojective $C^{*}$-algebra. A semiprojective $C^{*}$-algebra retains, in the noncommutative setting, enough of the properties of the algebra of continuous functions on an ANR to be used in a workable shape theory for $C^{*}$ algebras. Theorem $\mathrm{A}$ implies that, for $X$ one-dimensional, $C(X)$ is semiprojective. In fact, the homotopy lifting property in Theorem A, when restricted to injective inductive limits $\lim _{\longrightarrow} B_{n}$, is equivalent to semiprojectivity $([2,3.2])$.

Semiprojectivity is a noncommutative analog of being homotopically dominated by an ANR. Blackadar [1] introduced a property for 
$C^{*}$-algebras which is a noncommutative analog of a space being an ANR. He called this property semiprojectivity, but since both this and Effros and Kaminker's semiprojectivity are useful, we will call a $C^{*}$-algebra with Blackadar's property a noncommutative $A N R$. It is unknown whether $C(X)$ is a noncommutative ANR for $X$ onedimensional.

The homotopy lifting property, stated in Theorem $\mathrm{A}$ for $C(X)$, defines a class of $C^{*}$-algebras lying between the semiprojective $C^{*}$ algebras and the noncommutative ANR's. There is a nice characterization, in the finitely presented case, that a $C^{*}$-algebra will belong to this class if and only if it is defined by relations that are stable in the sense of the conclusions of Lemma 4.3, excluding (iii) and (v), (cf. notation 3.3). While a shape theory based on this class of $C^{*}$-algebras could be developed, it is not clear, at least at present, what advantages it would have over the two existing shape theories.

It may not be clear why Theorem $B$ is related to the rigidity property for maps to ANR's. This property is related to the concept of $\mathscr{U}$ closeness, which we now consider. Let $X$ and $Y$ be compact spaces and let $\varphi_{1}, \varphi_{2}: C(X) \rightarrow C(Y)$ be defined by functions $h_{1}, h_{2}: Y \rightarrow X$. If $\mathscr{U}$ is a finite open cover, then $h_{1}$ and $h_{2}$ are said to be $\mathscr{U}$-close if, for each $y \in Y$, there exists $U \in \mathscr{U}$ such that $h_{i}(y) \in U$ for $i=1,2$. Given $\varepsilon>0, f_{1}, \ldots, f_{k} \in C(X)$, there is a finite open cover $\mathscr{U}$ such that $h_{1}$ and $h_{2} \mathscr{U}$-close implies that

$$
\left\|\varphi_{1}\left(f_{i}\right)-\varphi_{2}\left(f_{i}\right)\right\|<\varepsilon, \quad i=1, \ldots, k .
$$

A converse also holds. If $X$ is a compact ANR, then it is a theorem $([5,1.3 .2])$ that there is a finite open cover $\mathscr{U}$ such that functions to $X$ which are $\mathscr{U}$-close are homotopic. Theorem B is precisely this statement, in the noncommutative setting, restricted to the onedimensional case.

The remaining sections are organized as follows. Detailed, elementary proofs of our main results are given in $\S 2$ for the simplest nontrivial case, i.e., the circle. These are intended to illustrate our general techniques involving generators and relations. A connected, onedimensional space is homotopic to a bouquet of circles $S^{1} \vee \cdots \vee S^{1}$. $\S 3$ describes the $C^{*}$-algebra $C\left(S^{1} \vee \cdots \vee S^{1}\right)$ in terms of generators and relations, and through a series of reductions shows that Theorems $A$ and $B$ follow from a lemma on the stability of these relations. The proof of this lemma, which contains the real work of this paper, is the content of $\S 4$. 
The author would like to thank the staff of MSRI for the wonderful working conditions provided there. He would also like to thank Edward Effros and Claude Schochet for helpful discussions on $C^{*}$ algebra homotopy.

2. A very special case. The key to proving Theorems $A$ and $B$ is Lemma 4.3. For $X=S^{1}$, Lemma 4.3 is vacuous. We shall prove Theorems A and B in this special case using some easy facts about the polar decomposition. There is nothing new or difficult in this section, but it serves to illustrate our approach for the more general case of $X=S^{1} \vee \cdots \vee S^{1}$.

If $X$ is an element of a $C^{*}$-algebra $A$, and if

$$
\left\|X^{*} X-1\right\|<1 \text { and }\left\|X X^{*}-1\right\|<1,
$$

then define $P(X)=X\left(X^{*} X\right)^{-1 / 2}$. The following properties of $P(X)$ are obvious:

(2.1) $P(X)$ is a unitary in $A$,

(2.2) $P$ is natural. I.e., if $\varphi: A \rightarrow B$ then $P(\varphi(X))=\varphi(P(X))$.

(2.3) If $X^{*} X=X X^{*}=1$ then $P(X)=X$.

Defining a homomorphism $\varphi: C\left(S^{1}\right) \rightarrow B$ is equivalent to specifying a unitary $U \in B$ as the image of $e^{2 \pi i x}$, where $e^{2 \pi i x}$ is the generating unitary of $C\left(S^{1}\right)$. If $B=\lim B_{n}$, then lifting $\varphi$ is equivalent to lifting $U$ to a unitary $V \in B_{N}$. By $(2.1)-(2.3)$ it suffices to lift $U$ to $X \in B_{N}$ with $\left\|X^{*} X-1\right\|$ and $\left\|X X^{*}-1\right\|$ small. More generally, we shall be interested in finding approximate lifts of operators satisfying relations to operators approximately satisfying those relations.

Lemma 2.1. Suppose $T_{1}, \ldots, T_{r}$ are elements of $B=\lim _{\longrightarrow} B_{n}$ and $p_{1}, \ldots, p_{k}$ are star-polynomials such that

$$
p_{i}\left(T_{1}, \ldots, T_{r}\right)=0, \quad i=1, \ldots, k .
$$

Let $\sigma_{n}: B_{n} \rightarrow B$ and $\sigma_{m, n}: B_{m} \rightarrow B_{n}$ denote the canonical maps defined by the connecting homomorphisms. For all $\varepsilon>0$ there exists $N$ and $S_{1}, \ldots, S_{r} \in B_{N}$ such that

$$
\left\|\sigma_{N}\left(S_{i}\right)-T_{i}\right\|<\varepsilon, \quad i=1, \ldots, r
$$

and

$$
\left\|p_{i}\left(S_{1}, \ldots, S_{r}\right)\right\|<\varepsilon, \quad i=1, \ldots, k
$$


Proof. This follows easily from the density of $\bigcup_{n=1, \ldots, \infty} \sigma_{n}\left(B_{n}\right)$ and the equality

$$
\left\|\sigma_{n}(X)\right\|=\lim _{m \rightarrow \infty}\left\|\sigma_{n, m}(X)\right\|
$$

for $X \in B_{n}$.

We now prove Theorem A for $X$ the circle. Suppose $\varphi: C\left(S^{1}\right) \rightarrow B$ and $B=\lim _{\longrightarrow} B_{n}$ with $\sigma_{n}: B_{n} \rightarrow B$ the canonical maps. Let $U=$ $\varphi\left(e^{2 \pi i x}\right)$. By Lemma 2.1, there exist $N$ and $X \in B_{N}$ such that

$$
\left\|X^{*} X-1\right\|<1, \quad\left\|X X^{*}-1\right\|<1
$$

and

$$
\left\|y_{t}^{*} y_{t}-1\right\|<1, \quad\left\|y_{t} y_{t}^{*}-1\right\|<1, \quad 0 \leq t \leq 1,
$$

where $y_{t}=t \sigma_{N}(X)+(1-t) U$.

Let $V=P(X), W_{t}=P\left(y_{t}\right)$. Property (2.1) shows that sending $e^{2 \pi i x}$ to $V$ (respectively $W_{t}$ ) defines a homomorphism $\bar{\varphi}: C\left(S^{1}\right) \rightarrow B_{N}$ (respectively $\varphi_{t}: C\left(S^{1}\right) \rightarrow B$ ). Property (2.2) shows that $\sigma_{N}(V)=W_{1}$, hence $\sigma_{N} \circ \bar{\varphi}=\varphi_{1}$, and (2.3) shows $W_{0}=U$, hence $\varphi_{0}=\varphi$. Therefore $\bar{\varphi}$ is a lift of $\varphi$ up to homotopy.

For $X=S^{1}$, Theorem B is true with $\varepsilon=2$ and $f_{0}=e^{2 \pi i x}$. This is because, given $\varphi_{0}, \varphi_{1}: C\left(S^{1}\right) \rightarrow A$, the path of unitaries

$$
P\left(t \varphi_{0}\left(e^{2 \pi i x}\right)+(1-t) \varphi_{1}\left(e^{2 \pi i x}\right)\right)
$$

defines a path of homomorphisms from $\varphi_{0}$ to $\varphi_{1}$ whenever $\| \varphi_{1}\left(e^{2 \pi i x}\right)-$ $\varphi_{2}\left(e^{2 \pi i x}\right) \|<2$.

3. The proofs. Our plan of attack is first to show that it suffices to prove Theorems A and B in the case $X=S^{1} \vee \cdots \vee S^{1}$, a bouquet of circles. We will describe the algebra $C\left(S^{1} \vee \cdots \vee S^{1}\right)$ in terms of generators and relations. Lemma 4.3 describes a construction which perturbs operators which almost satisfy these relations so that they satisfy them exactly. With this construction we prove our results for $X=S^{1} \vee \cdots \vee S^{1}$.

Every one-dimensional finite CW-complex is homotopy-equivalent to the disjoint union of a finite number of finite bouquets of circles. The reduction to the case $X=S^{1} \vee \cdots \vee S^{1}$ therefore follows from the next two propositions. 
PROPOSITION 3.1. If Theorem A (respectively B) holds for $X$, and if $Y$ is homotopically dominated by $X$, then Theorem A (respectively B) holds for $Y$.

Proof. For Theorem A, the proof is exactly as for [2, 3.4].

Suppose $\alpha: C(X) \rightarrow C(Y), \beta: C(Y) \rightarrow C(X)$ and $\alpha \circ \beta \sim 1$. Suppose also that the conclusion of Theorem $B$ holds for $\varepsilon>0$ and $f_{1}, \ldots, f_{k} \in C(X)$. If $\varphi_{1}, \varphi_{2}: C(Y) \rightarrow B$ satisfy the condition

$$
\left\|\varphi_{1}\left(\alpha\left(f_{i}\right)\right)-\varphi_{2}\left(\alpha\left(f_{i}\right)\right)\right\|<\varepsilon, \quad i=1, \ldots, k,
$$

then $\varphi_{1} \circ \alpha \sim \varphi_{2} \circ \alpha$ hence

$$
\varphi_{1} \sim \varphi_{1} \circ \alpha \circ \beta \sim \varphi_{2} \circ \alpha \circ \beta \sim \varphi_{2},
$$

which shows that the conclusions of Theorem B also hold for $\varepsilon$ and $\alpha\left(f_{1}\right), \ldots, \alpha\left(f_{k}\right) \in C(Y)$.

Proposition 3.2. If Theorem $\mathrm{A}$ (respectively B) holds for $X_{1}$ and $X_{2}$, then Theorem A (respectively B) holds for their disjoint union $X_{1} \cup X_{2}$.

Proof. For Theorem A, the proof of this proposition is similar to that of $[2,3.6]$ and is omitted.

We identify $C\left(X_{1}\right) \oplus C\left(X_{2}\right)$ with $C\left(X_{1} \cup X_{2}\right)$ and $C\left(X_{i}\right)$ with $C\left(X_{1}\right) \oplus$ 0 or $0 \oplus C\left(X_{2}\right)$. Choose $\varepsilon_{i}>0, f_{1}^{i}, \ldots, f_{n_{l}}^{i} \in C\left(X_{i}\right)$ such that the conclusion of Theorem B holds for $\varepsilon_{i},\left\{f_{1}^{i}, \ldots, f_{n_{l}}^{i}\right\}$. Without loss of generality, assume that $\varepsilon_{1}=\varepsilon_{2}=\varepsilon, n_{1}=n_{2}=n$ and $\left\|f_{j}^{i}\right\| \leq 1$. We shall show that Theorem B holds for $X_{1} \cup X_{2}$ with $\varepsilon_{0}$ and

$$
\{1 \oplus 0,0 \oplus 1\} \cup\left\{f_{j}^{i} \mid i=1,2, j=1, \ldots, n\right\}
$$

where $\varepsilon_{0}$ is chosen below.

Lemma 1.8 of [3] implies that there exists $\delta>0$ such that, given two pairs of orthogonal projections $\left\{E_{1}, E_{2}\right\},\left\{F_{1}, F_{2}\right\}$ in a $C^{*}$-algebra $A$, if $\left\|E_{i}-F_{i}\right\|<\delta, i=1,2$, then there exists a unitary $U \in A$ such that

$$
\|U-1\|<\min \{\varepsilon / 3,1\}
$$

and

$$
U E_{i} U^{*}=F_{i}, \quad i=1,2 .
$$

We let $\varepsilon_{0}=\min \{\delta, \varepsilon / 3\}$.

Assume that

$$
\varphi, \psi: C\left(X_{1}\right) \oplus C\left(X_{2}\right) \rightarrow B
$$


are given, and let $p_{1}=\varphi(1 \oplus 0), p_{2}=\varphi(0 \oplus 1), q_{1}=\psi(1 \oplus 0)$, $q_{2}=\psi(0 \oplus 1)$. Assume further that

$$
\begin{gathered}
\left\|\varphi\left(f_{j}^{i}\right)-\psi\left(f_{j}^{i}\right)\right\|<\varepsilon_{0}, \quad i=1,2, j=1, \ldots, n, \\
\left\|p_{i}-q_{i}\right\|<\varepsilon_{0}, \quad i=1,2 .
\end{gathered}
$$

Because $\varepsilon_{0}<\delta$, there exists a unitary $U \in B$ with

$$
\|U-1\|<\min \{\varepsilon / 3,1\}
$$

and

$$
U q_{i} U^{*}=p_{i}, \quad i=1,2 .
$$

Since $\|U-1\|<1, \psi$ is homotopic to $\operatorname{Ad}_{U} \circ \psi$. Therefore, it suffices to show that the (unital) homomorphisms

$$
\left.\varphi\right|_{C\left(X_{i}\right)},\left.\operatorname{Ad}_{U} \circ \psi\right|_{C\left(X_{i}\right)}: C\left(X_{i}\right) \rightarrow p_{i} B p_{i}
$$

are homotopic for $i=1,2$.

For $f=f_{j}^{i}$,

$$
\begin{aligned}
\| \varphi(f) & -\operatorname{Ad}_{U} \circ \psi(f) \| \\
& \leq\|\varphi(f)-\psi(f)\|+\|\psi(f)-U \psi(f)\|+\left\|U \psi(f)-U \psi(f) U^{*}\right\| \\
& \leq\|\varphi(f)-\psi(f)\|+2\|U-1\|<\varepsilon_{0}+2 \varepsilon / 3<\varepsilon .
\end{aligned}
$$

By our original assumptions, this implies that $\left.\varphi\right|_{C\left(X_{i}\right)}$ and $\left.\operatorname{Ad}_{U} \circ \psi\right|_{C\left(X_{i}\right)}$ are homotopic.

We now consider the case $X=S^{1} \vee \cdots \vee S^{1}$ ( $N$ copies). Since $X$ sits naturally as a closed subset of the $N$-torus, it is clear that $C(X)$ is generated by $N$ commuting unitaries. It is not hard to discover the relations satisfied by these unitaries.

Notation 3.3. If $V_{1}, \ldots, V_{N}$ are unitaries in a $C^{*}$-algebra, we let

$$
\mathscr{E}\left(V_{1}, \ldots, V_{N}\right)=\max _{i \neq j}\left\|\left(1-V_{i}\right)\left(1-V_{j}\right)\right\| .
$$

Proposition 3.4. The $C^{*}$-algebra $C\left(S^{1} \vee \cdots \vee S^{1}\right)$ is the universal $C^{*}$ algebra generated by $N$ unitaries $v_{1}, \ldots, v_{N}$ such that $\mathscr{E}\left(v_{1}, \ldots, v_{N}\right)=$ 0 . If $S^{1} \vee \cdots \vee S^{1}$ is realized as $\{1, \ldots, N\} \times[0,1] / \sim$, where $\sim$ identifies all endpoints, then the canonical generators are

$$
v_{j}(k, t)= \begin{cases}e^{2 \pi i t} & \text { if } j=k, \\ 1 & \text { if } j \neq k .\end{cases}
$$


Proof. Let $D$ denote the universal $C^{*}$-algebra generated by $N$ unitaries subject to $\mathscr{E}\left(v_{1}, \ldots, v_{N}\right)=0$. The relation

$$
\left(1-v_{i}\right)\left(1-v_{j}\right)=\left(1-v_{j}\right)\left(1-v_{i}\right)
$$

implies that $D$ is commutative, and so must be a quotient of $C\left(\mathbf{T}^{N}\right)$. More precisely, $D \cong C(Z)$ where $Z \subseteq \mathbf{T}^{N}$ is the intersection of the zero-sets of the functions

$$
\left(z_{1}, \ldots, z_{n}\right) \rightarrow\left(1-z_{i}\right)\left(1-z_{j}\right), \quad z_{k} \in \mathbf{T} \subseteq \mathbf{C},
$$

for $i \neq j$. I.e., $Z$ is the set of points $\left(z_{1}, \ldots, z_{N}\right) \in \mathbf{T}^{N} \subseteq \mathbf{C}^{N}$ where at most one coordinate is not equal to 1 .

Our proofs of Theorems A and B for $X=S^{1} \vee \cdots \vee S^{1}$ require that, for a sufficiently small $\varepsilon>0$, there should exist a mapping $\left(V_{1}, \ldots, V_{N}\right) \rightarrow\left(\tilde{V}_{1}, \ldots, \tilde{V}_{N}\right)$ of $N$-tuples of unitaries as follows. Whenever $V_{1}, \ldots, V_{N}$ are unitaries in $A$ such that $\mathscr{E}\left(V_{1}, \ldots, V_{N}\right)<\varepsilon$, an $N$-tuple $\left(\tilde{V}_{1}, \ldots, \tilde{V}_{N}\right)$ of unitaries in $A$ should be defined so that the following properties hold:

(3.1) $\mathscr{E}\left(\tilde{V}_{1}, \ldots, \tilde{V}_{N}\right)=0$.

(3.2) $\left(V_{1}, \ldots, V_{N}\right) \rightarrow\left(\tilde{V}_{1}, \ldots, \tilde{V}_{N}\right)$ is natural.

(3.3) If $\mathscr{E}\left(V_{1}, \ldots, V_{N}\right)=0$, there exist continuous paths $V_{i}^{(t)}$

of unitaries from $V_{i}$ to $\tilde{V}_{i}$ with $\mathscr{E}\left(V_{1}^{(t)}, \ldots, V_{N}^{(t)}\right)=0$.

The main point of Lemma 4.3 is that such a mapping exists. Let us now see how, for $X=S^{1} \vee \cdots \vee S^{1}$, Theorems A and B follow from this. Fix an $\varepsilon>0$ for which this mapping exists.

Suppose $\varphi, \psi: C\left(S^{1} \vee \cdots \vee S^{1}\right) \rightarrow B$. Choose $\eta>0$ such that

$$
\left\|\varphi\left(v_{i}\right)-\psi\left(v_{i}\right)\right\|<\eta, \quad i=1, \ldots, N,
$$

implies that $\mathscr{E}\left(W_{1, s}, \ldots, W_{N, s}\right)<\varepsilon$, where

$$
W_{i, s}=P\left(s \varphi\left(v_{i}\right)+(1-s) \psi\left(v_{i}\right)\right) .
$$

Here $P(X)=X\left(X^{*} X\right)^{-1 / 2}$ as in $\S 2$. Properties (3.1) and (3.2) imply that $v_{i} \rightarrow \tilde{W}_{i, s}$ defines a continuous path of homomorphisms $\alpha_{s}$ : $C\left(S^{1} \vee \cdots \vee S^{1}\right) \rightarrow B$ whenever (3.4) holds. Since $W_{i, 1}=\varphi\left(v_{i}\right)$ and $W_{i, 0}=\psi\left(v_{i}\right)$, property (3.3) implies $\varphi \sim \alpha_{1} \sim \alpha_{0} \sim \psi$. This proves Theorem B in this case.

Suppose $\varphi: C\left(S^{1} \vee \cdots \vee S^{1}\right) \rightarrow B$ is given, with $B=\lim B_{n}$ and $\sigma_{n}: B_{n} \rightarrow B$ the canonical maps. Applying Lemma 2.1 to $\vec{\varphi}\left(v_{1}\right), \ldots$, $\varphi\left(v_{N}\right)$ produces elements $X_{1}, \ldots, X_{N} \in B_{k}$ with $\left\|\left(1-X_{i}\right)\left(1-X_{j}\right)\right\|$ and 
$\left\|\sigma_{k}\left(X_{i}\right)-\varphi\left(v_{i}\right)\right\|$ small. Consequently, if we define

$$
W_{i}=P\left(X_{i}\right), \quad U_{i, s}=P\left(s \sigma_{k}\left(X_{i}\right)+(1-s) \varphi\left(v_{i}\right)\right),
$$

$\mathscr{E}\left(W_{1}, \ldots, W_{N}\right)$ and $\mathscr{E}\left(U_{1, s}, \ldots, U_{N, s}\right)$ can be assumed to be small enough so that $\tilde{W}_{i}$ and $\tilde{U}_{i, s}$ are defined. These define homomorphisms

$$
\bar{\varphi}: C\left(S^{1} \vee \cdots \vee S^{1}\right) \rightarrow B_{k} \quad \text { and } \quad \alpha_{s}: C\left(S^{1} \vee \cdots \vee S^{1}\right) \rightarrow B .
$$

Property (3.2) implies $\sigma_{k}\left(\tilde{W}_{i}\right)=\tilde{U}_{i, 1}$ hence $\sigma_{k} \bar{\varphi}=\alpha_{1}$. But $\alpha_{0} \sim \varphi$ by property (3.3), so $\bar{\varphi}$ is a lift of $\varphi$ up to homotopy, proving Theorem $A$ in this case.

4. Stability of the relations. This section is devoted to finding a natural method of perturbing unitaries $V_{1}, \ldots, V_{N}$ for which the relations

$$
\left(1-V_{i}\right)\left(1-V_{j}\right)=0, \quad i \neq j,
$$

almost hold into ones for which (4.1) holds exactly. The construction proceeds iteratively, perturbing at each step only the first $k$ unitaries to force (4.1) to hold for $i, j \leq k$. In order to control the error in the remaining relations, we keep track of the size of the perturbations. As a result, Lemma 4.3 is stronger than the proofs of Theorems A and B require.

Since we will use the polar decomposition $P(X)=X\left(X^{*} X\right)^{-1 / 2}$, we need the following estimate.

Lemma 4.1. Suppose $U, V \in \mathscr{B}(\mathscr{H})$ and $U$ is unitary and $\|U-V\| \leq$ $C \leq 1 / 12$. Then $\|U-P(V)\| \leq 7 C$.

Proof. The spectral mapping theorem and a little calculus show that $\left\|h^{-1 / 2}-1\right\| \leq \Delta$ whenever $h>0$ and $\|h-1\| \leq \Delta \leq 1 / 4$. Trivial estimates show that

$$
\|U-P(V)\| \leq C+2\left\|\left(V^{*} V\right)^{-1 / 2}-1\right\|
$$

and $\left\|V^{*} V-1\right\| \leq 3 C$, so the required estimate follows immediately.

The next lemma shows that, once the first $n$ unitaries have been perturbed to satisfy (4.1), the first $n+1$ can be perturbed slightly so that (4.1) holds for $i, j \leq n+1$.

Recall, from notation 3.3 , that $\mathscr{E}\left(V_{1}, \ldots, V_{N}\right)$ measures the error in (4.1) for unitaries $V_{1}, \ldots, V_{N}$.

LEMMA 4.2. Let $n$ be a positive integer, $\delta$ a real number $0<\delta \leq 1 / 4$, and $A$ a $C^{*}$-algebra. Given an $(n+1)$-tuple $\left(V_{1}, \ldots, V_{n}, U\right)$ of unitaries 
in $A$ such that

(i) $\mathscr{E}\left(V_{1}, \ldots, V_{n}\right)=0$,

(ii) $\mathscr{E}\left(V_{1}, \ldots, V_{n}, U\right)<\delta / 12 \sqrt{2} n$, there is a natural assignment of unitaries $\left(V_{1}^{\delta}, \ldots, V_{n}^{\delta}, U^{\delta}\right)$ in $A$ such that

(iii) $\mathscr{E}\left(V_{1}^{\delta}, \ldots, V_{n}^{\delta}, U^{\delta}\right)=0$,

(iv) $\left\|U-U^{\delta}\right\| \leq 84 \sqrt{2} n \mathscr{E}\left(V_{1}, \ldots, V_{n}, U\right) / \delta$,

(v) $\left\|V_{i}-V_{i}^{\delta}\right\| \leq \delta / \pi, i=1, \ldots, n$,

(vi) if $\mathscr{E}\left(V_{1}, \ldots, V_{n}, U\right)=0$ then there exist natural paths $V_{i}^{\delta, t}, U^{\delta, t}$ of unitaries from $V_{i}, U$ to $V_{i}^{\delta}, U^{\delta}$ such that $\mathscr{E}\left(V_{1}^{\delta, t}, \ldots, V_{n}^{\delta, t}, U^{\delta, t}\right)=0$,

(vii) if $V_{1}=\cdots=V_{n}=U=1$ then $V_{1}^{\delta, t}=\cdots=V_{n}^{\delta, t}=U^{\delta, t}=1$.

Proof. We shall use the notation and description of $S^{1} \vee \cdots \vee S^{1}$ given in Proposition 3.4. Suppose $\left(V_{1}, \ldots, V_{n}, U\right) \in \mathscr{U}(A)^{n+1}$ satisfy (i) and (ii). By Proposition 3.4, sending $v_{i}$ to $V_{i}$ defines a homomorphism $\rho: C\left(S^{1} \vee \cdots \vee S^{1}\right) \rightarrow A$. We will abuse notation and identify $\rho(g)$ with $g$ for any $g \in C\left(S^{1} \vee \cdots \vee S^{1}\right)$, and so $v_{i}$ with $V_{i}$.

Let $f$ denote the function which takes the value 1 on the intervals $\{l\} \times[2 \delta, 1-2 \delta]$, the value 0 on the complement of the intervals $\{l\} \times[\delta, 1-\delta]$, and is linear in between.

Define $U^{\prime}$ and $U^{\delta}$ by

$$
U^{\prime}=f+\sqrt{1-f} U \sqrt{1-f}, \quad U^{\delta}=P\left(U^{\prime}\right),
$$

and define $V_{k}^{\delta}$ by

$V_{k}^{\delta}(l, t)=\left\{\begin{array}{cl}\exp (2 \pi i(t-2 \delta) /(1-4 \delta)) & \text { if } l=k \text { and } t \in[2 \delta, 1-2 \delta] \\ 1 & \text { otherwise. }\end{array}\right.$

It is not yet evident that $U^{\prime}$ is invertible. However, assuming this to be true, it is clear that $U^{\delta}, V_{k}^{\delta}$ are unitary and the construction is natural. Also, (v) is obvious. Assuming (iv), it is easy to see that the paths $V_{k}^{t \delta}, U$ satisfy (vi) and (vii). (For (vii), note that $\rho(g)=g(l, 0) 1$ when $V_{1}=\cdots=V_{n}=U=1$.) Therefore, to finish the proof, we must show that $U^{\prime}$ is invertible and prove (iii) and (iv).

Since $\left(1-V_{k}^{\delta}\right) f=\left(1-V_{k}^{\delta}\right)$ and $\left(1-V_{k}^{\delta}\right) \sqrt{1-f}=0$, we see that $\left(1-V_{k}^{\delta}\right) U^{\prime}=\left(1-V_{k}^{\delta}\right) U^{\prime *}=\left(1-V_{k}^{\delta}\right)$. By approximating $\left(U^{\prime *} U^{\prime}\right)^{-1 / 2}$ by polynomials in $U^{* *} U^{\prime}$, one finds that $\left(1-V_{k}^{\delta}\right) U^{\delta}=\left(1-V_{k}^{\delta}\right)$. Therefore (iii) is true.

Our estimate of $\left\|U-U^{\prime}\right\|$ will rest on the following. Suppose $g \in$ $C\left(S^{1} \vee \cdots \vee S^{1}\right)$ satisfies $\|g\| \leq 1$ and $g(l, t)=0$ if $t \notin[\delta, 1-\delta]$. Define

$$
h_{k}(l, t)=\left\{\begin{array}{cl}
(g(l, t)) /\left(e^{2 \pi i t}-1\right) & \text { if } l=k \text { and } t \in[\delta, 1-\delta], \\
0 & \text { otherwise. }
\end{array}\right.
$$


Then $\left\|h_{k}\right\| \leq 4 \sqrt{2} / \delta$ and

$$
g=\sum_{k}\left(V_{k}-1\right) h_{k}
$$

Therefore,

$$
\begin{aligned}
\|(U-1) g\| & \leq \sum_{k}\left\|(U-1)\left(V_{k}-1\right)\right\|\left\|h_{k}\right\| \\
& \leq 4 \sqrt{2} n \mathscr{E}\left(V_{1}, \ldots, V_{n}, U\right) / \delta
\end{aligned}
$$

and the same estimate holds for $\|g(U-1)\|$.

The equality

$$
\begin{aligned}
U-U^{\prime}= & (U-1) f \\
& +[(U-1)(\sqrt{1-f}-1)-(\sqrt{1-f}-1)(U-1)] \sqrt{1-f}
\end{aligned}
$$

implies the inequality

$$
\begin{aligned}
\left\|U-U^{\prime}\right\| \leq & \|(U-1) f\|+\|(U-1)(\sqrt{1-f}-1)\| \\
& +\|(\sqrt{1-f}-1)(U-1)\| .
\end{aligned}
$$

By (4.2), with $g=f$ or $g=\sqrt{1-f}-1$, this implies

$$
\left\|U-U^{\prime}\right\| \leq 12 \sqrt{2} n \mathscr{E}\left(V_{1}, \ldots, V_{n}, U\right) / \delta .
$$

Thus $U^{\prime}$ is invertible, and by Lemma 4.1, (v) holds.

As previously remarked, Lemma 4.3 is stronger than is needed to prove Theorems A and B. Conditions (iii), (v), and the naturality of the paths $V_{k}^{(t)}$ were added to allow a proof by induction. Condition (iii) may, however, prove to be of interest.

LEMMA 4.3. For any $\varepsilon>0$ and any integer $N>0$, there exists $\eta>0$ such that, given an $N$-tuple of unitaries $\left(V_{1}, \ldots, V_{N}\right)$ in a $C^{*}$-algebra A satisfying

(i) $\mathscr{E}\left(V_{1}, \ldots, V_{N}\right)<\eta$,

there is a natural assignment of unitaries $\left(\tilde{V}_{1}, \ldots, \tilde{V}_{N}\right)$ in $A$ such that

(ii) $\mathscr{E}\left(\tilde{V}_{1}, \ldots, \tilde{V}_{N}\right)=0$,

(iii) $\left\|\tilde{V}_{k}-V_{k}\right\|<\varepsilon$ for all $k$,

(iv) if $\mathscr{E}\left(V_{1}, \ldots, V_{N}\right)=0$ then there are natural, continuous paths $V_{k}^{(t)}$ of unitaries from $V_{k}$ to $\tilde{V}_{k}$ such that $\mathscr{E}\left(V_{1}^{(t)}, \ldots, V_{N}^{(t)}\right)=0$,

(v) if $V_{1}=\cdots=V_{N}=1$, then $V_{1}^{(t)}=\cdots=V_{N}^{(t)}=1$. 
Proof. We shall proceed by induction on the number of unitaries. Since $\mathscr{E}(U)=0$ for all unitaries $U$, the lemma is completely trivial for $N=1$.

Assume the lemma is true for $N-1$ unitaries. Let $\varepsilon>0$ be given, and choose $\delta>0$ so that

$$
\begin{gathered}
\delta<1 / 4 \\
\delta / \pi<\varepsilon / 2
\end{gathered}
$$

Choose $\varepsilon_{0}>0$ so that

$$
\begin{gathered}
84 \sqrt{2}(N-1) \varepsilon_{0} / \delta<\varepsilon / 3 \\
\varepsilon_{0}<\varepsilon / 2 \\
\varepsilon_{0}<\delta / 36 \sqrt{2}(N-1) .
\end{gathered}
$$

We now use the induction hypothesis, with $\varepsilon_{0}$ and $N-1$ in place of $\varepsilon$ and $N$, to produce $\eta_{0}>0$ for which (ii) $-(v)$ can be fulfilled. Finally, choose $\eta>0$ so that

$$
\begin{gathered}
\eta<\eta_{0}, \\
\eta<\delta / 36 \sqrt{2}(N-1), \\
84 \sqrt{2}(N-1) \eta / \delta<\varepsilon / 3 .
\end{gathered}
$$

Suppose that $\mathscr{E}\left(V_{1}, \ldots, V_{N}\right)<\eta$. By (4.8) and the induction hypothesis, there is a natural choice $\left(\bar{V}_{1}, \ldots, \bar{V}_{N-1}\right)$ of unitaries such that

$$
\begin{gathered}
\mathscr{E}\left(\bar{V}_{1}, \ldots, \bar{V}_{N-1}\right)=0, \\
\left\|\bar{V}_{k}-V_{k}\right\|<\varepsilon_{0} .
\end{gathered}
$$

If $\mathscr{E}\left(V_{1}, \ldots, V_{N}\right)=0$, then by assumption there are natural paths of unitaries $\bar{V}_{k, t}, k<N$, from $V_{k}$ to $\bar{V}_{k}$, such that $\mathscr{E}\left(\bar{V}_{1, t}, \ldots, \bar{V}_{N-1, t}\right)=$ 0 . Later, we will need to know that

$$
\mathscr{E}\left(\bar{V}_{1, t}, \ldots, \bar{V}_{N-1, t}, V_{N}\right)=0 .
$$

To prove this, assume $A \subseteq \mathscr{B}(\mathscr{H})$. Let $p$ be the spectral projection of $V_{N}$ for the set $\{z \in \mathrm{C}|| z \mid=1, z \neq 1\}$. If $p=0$ then (4.13) is trivial, so assume that $p \neq 0$. By writing $p$ as the weak limit of positive operators in $A$ and using Proposition 3.4, it can be shown that $p\left(1-V_{k}\right) p=0$ for $k<N$. (Note that $p$ corresponds to the characteristic function of the set $\{N\} \times(0,1)$.) Thus $V_{k}$ is in the $C^{*}$-algebra $p \mathbf{C} \times(1-p) \mathscr{B}(\mathscr{H})(1-p)$ and is sent to 1 under the projection of this $C^{*}$-algebra onto $p \mathbf{C} \cong \mathbf{C}$. 
Condition ( $\mathrm{v}$ ) in the induction hypothesis and naturality imply that

$$
\bar{V}_{k, t}=p+(1-p) \bar{V}_{k, t}(1-p) \text {. }
$$

By our choice of $p$,

$$
\begin{aligned}
\left(1-V_{N}\right)\left(1-\bar{V}_{k, t}\right) & =\left[p\left(1-V_{N}\right) p\right]\left[(1-p)\left(1-\bar{V}_{k, t}\right)(1-p)\right]=0 \\
& =\left(1-\bar{V}_{k, t}\right)\left(1-V_{N}\right),
\end{aligned}
$$

proving (4.13).

Returning to the general case of $\mathscr{E}\left(V_{1}, \ldots, V_{N}\right)<\eta$, we have, for any $k<N$,

$$
\begin{aligned}
\left\|\left(1-V_{N}\right)\left(1-\bar{V}_{k}\right)\right\| & \leq\left\|\left(1-V_{N}\right)\left(1-V_{K}\right)\right\|+\left\|\left(1-V_{N}\right)\left(V_{k}-\bar{V}_{k}\right)\right\| \\
& \leq \eta+2\left\|V_{k}-\bar{V}_{k}\right\| \leq \eta+2 \varepsilon_{0} .
\end{aligned}
$$

A similar estimate for the reverse product, together with (4.11), shows

$$
\mathscr{E}\left(\bar{V}_{1}, \ldots, \bar{V}_{N-1}, V_{N}\right) \leq \eta+2 \varepsilon_{0} \text {. }
$$

Therefore, we may apply Lemma 4.2 , by (4.7) and (4.9), and conclude that there is a natural choice $\left(\tilde{V}_{1}, \ldots, \tilde{V}_{N}\right)=\left(\bar{V}_{1}^{\delta}, \ldots, \bar{V}_{N-1}^{\delta}, V_{N}^{\delta}\right)$ such that

$$
\begin{aligned}
& \mathscr{E}\left(\tilde{V}_{1}, \ldots, \tilde{V}_{N}\right)=0, \quad\left\|V_{N}-\tilde{V}_{N}\right\| \leq 84 \sqrt{2}(N-1)\left(\eta+2 \varepsilon_{0}\right) / \delta, \\
& \left\|V_{k}-\tilde{V}_{k}\right\| \leq\left\|V_{k}-\bar{V}_{k}\right\|+\left\|\bar{V}_{k}-\tilde{V}_{k}\right\| \leq \varepsilon_{0}+\delta / \pi, \quad k<N .
\end{aligned}
$$

Since $\left\|V_{N}-\tilde{V}_{N}\right\|<\varepsilon$, by (4.5) and (4.10), and $\left\|V_{k}-\tilde{V}_{k}\right\|<\varepsilon, k<N$, by (4.4) and (4.6), we have proven (ii) and (iii).

If $\mathscr{E}\left(V_{1}, \ldots, V_{N}\right)=0$ then the concatenation of the paths

$$
t \rightarrow \bar{V}_{k}^{\delta, t}, \quad k \leq N
$$

and the paths

$$
\begin{aligned}
& t \rightarrow \bar{V}_{k, t}, \quad k<N, \\
& t \rightarrow V_{N}
\end{aligned}
$$

satisfy (iv) and (v).

\section{REFERENCES}

[1] B. Blackadar, Shape theory for $C^{*}$-algebras, Math. Scand., 56 (1985), 249-275.

[2] E. Effros and J. Kaminker, Homotopy continuity and shape theory for $C^{*}$. algebras, in Geometric Methods in Operator Algebras, U.S.-Japan Joint Seminar at Kyoto, 1983, Pitman, to appear. 
[3] J. Glimm, On a certain class of operator algebras, Trans. Amer. Math. Soc., 95 (1960), 318-340.

[4] T. A. Loring, The $K$-theory of $A F$ embeddings of the rational rotation algebras, $K$-theory, to appear.

[5] S. Mardesic and J. Segal, Shape Theory, North-Holland, Amsterdam (1982).

Received August 25, 1987 and in revised form February 28, 1988. This research was conducted at the Mathematical Sciences Research Institute, supported by NSF grant MCS-812079-05, and at the University of Warwick, supported by the SERC.

UNIVERSITY OF WARWICK

COVENTRY CV4 7AL, ENGLAND

Current address: Dalhousie University

Halifax, Nova Scotia B3H 3J5

Canada 


\section{PACIFIC JOURNAL OF MATHEMATICS EDITORS}

\author{
V. S. VARADARAJAN \\ (Managing Editor) \\ University of California \\ Los Angeles, CA 90024 \\ HeRbert Clemens \\ University of Utah \\ Salt Lake City, UT 84112 \\ THOMAS ENRIGHT \\ University of California, San Diego \\ La Jolla, CA 92093
}

R. FINN

Stanford University

Stanford, CA 94305

HERMANN FLASCHKA

University of Arizona

Tucson, AZ 85721

VAUGHAN F. R. JONES

University of California

Berkeley, CA 94720

STEVEN KERCKHOFF

Stanford University

Stanford, CA 94305

\section{ROBION KIRBY}

University of California

Berkeley, CA 94720

C. C. MOORE

University of California

Berkeley, CA 94720

HAROLD STARK

University of California, San Diego

La Jolla, CA 92093

\section{ASSOCIATE EDITORS}
R. ARenS
E. F. BECKENBACH
B. H. NeumanN
F. WOLF
K. YOSHIDA (1906-1982)

\section{SUPPORTING INSTITUTIONS}
UNIVERSITY OF ARIZONA
UNIVERSITY OF OREGON
UNIVERSITY OF BRITISH COLUMBIA
UNIVERSITY OF SOUTHERN CALIFORNIA
CALIFORNIA INSTITUTE OF TECHNOLOGY
STANFORD UNIVERSITY
UNIVERSITY OF CALIFORNIA
MONTANA STATE UNIVERSITY
UNIVERSITY OF HAWAII
UNIVERSITY OF NEVADA, RENO
UNIVERSITY OF TOKYO
NEW MEXICO STATE UNIVERSITY
UNIVERSITY OF UTAH
OREGON STATE UNIVERSITY
WASHINGTON STATE UNIVERSITY
UNIVERSITY OF WASHINGTON 


\section{Pacific Journal of Mathematics}

\section{Vol. 136, No. $1 \quad$ November, 1989}

Robert Archbold and Frederic W. Shultz, Characterization of $C^{*}$-algebras with continuous trace by properties of their pure states $\ldots \ldots \ldots \ldots \ldots \ldots 1$

Shu Ping Chen and Roberto Triggiani, Proof of extensions of two conjectures on structural damping for elastic systems $\ldots \ldots \ldots \ldots \ldots \ldots$

Philip Throop Church and James Timourian, A nonlinear elliptic

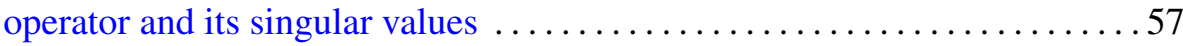

A. Gervasio Colares and Katsuei Kenmotsu, Isometric deformation of surfaces in $R^{3}$ preserving the mean curvature function $\ldots \ldots \ldots \ldots \ldots 71$

Fei Xu, A remark on spinor norms of local integral rotations. I . . . . . . . 81

Pedro Martinez Gadea and Ángel María Montesinos-Amilibia, Spaces of constant para-holomorphic sectional curvature $\ldots \ldots \ldots \ldots \ldots \ldots \ldots 5$

Guangxin Zeng, Homogeneous Stellensätze in semialgebraic geometry . . . . 103

Thomas Eric Hall, The isomorphism problem for orthodox semigroups . . . . 123

Mike Hoffman, Noncoincidence index, free group actions, and the fixed

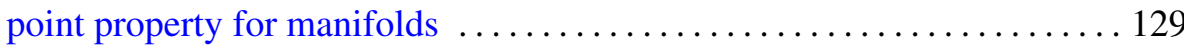

Terry Atherton Loring, The noncommutative topology of one-dimensional

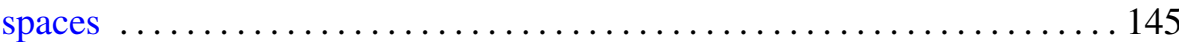

Haskell Paul Rosenthal and Alan Evan Wessel, The Krě̆ n-Mil'man property and a martingale coordinatization of certain nondentable convex sets

Yoshimi Saito, A remark on the limiting absorption principle for the reduced wave equation with two unbounded media 